\title{
A Review: Towards Quality of Service in Cloud Computing
}

\author{
Juhi Singh $^{1}$, Shalini Agarwal ${ }^{2}$, Jayant Mishra ${ }^{3}$ \\ ${ }^{1,3}$ Department of Computer Science and Engineering, SRMGPC, Lucknow \\ ${ }^{2}$ Department of Computer Science and Engineering, SRMU, Lucknow
}

\begin{abstract}
Quality of Service (QoS) plays a vital role in the reservation of resources within service oriented distributed systems and has been widely worked on well established paradigm of Grid Computing. Cloud Computing using the concept of virtualization creates new challenges for improvement in Quality of Service. Today Cloud Computing is emerging as most widespread technology in every field of data storage and resource sharing over the network. Getting into this new emerging technology, quality performance measures need to be improved for the ease of data availability, resource utilization, cost, response time, restoration and number of parameters. The paper emphasizes on the research gap for the improvement of Quality of Services in Cloud Computing. In all the paper enlighten the researches that has been done relevant to QoS in Cloud Computing and future research is proposed.
\end{abstract}

Keywords: Cloud Computing, Grid Computing, Quality of Service (QoS), Virtual Machine (VM), Virtualization

\section{Introduction}

Quality-of-Service (QoS) in cloud computing is defined in terms of allocating resources to the application that guaranties a service level along dimensions such as performance, availability and reliability. Earlier QoS has been an issue in many of the Distributed Computing technology as Grid Computing. The purpose of this paper is to address QoS especially in the reference of Cloud Computing and its related research gap. The paper discusses the QoS parameters and the term virtualization in Cloud Computing. The paper also depicts a brief study done on QoS provisioning in Grids. And as we move towards the technology to enable it in Cloud Computing QoS need to be improved through various algorithms for cost optimization as partial critical path [1], resource management techniques [2][3].

Various techniques are used to improve the quality of service over cloud computing. Virtualization technique of cloud computing has moved its steps towards optimized resource utilization and methods to access data stored with high availability and restoration [4][5]. Various work has been done for quality improvement and security of data over the cloud computing [6][7]. The paper provides a direction about early research works on Quality of Service in cloud environment, that categorize the contributions according to relevant areas and methods used. [8][9]. Most of the present day organizations have moved to cloud computing for the data storage. Even largest online retailers with a number of operations in world's leading countries and wide spreading operations across the globe depend on cloud services. The ecommerce has emerged as a enlarged role in the cloud computing space with its Amazon Web Services (AWS)[10][11][12][13]. Commercial fields are also one of the leading providers of on-demand Customer Relationship Management (CRM) softwares which enables organizations for the better management of secured operations and inhabits services such as sales force automation, customer service and support, marketing automation, document management, analytics and custom application development. Other most widely used web-based portal Facebook Inc. creates social networks that has large and continuous access of heavy data. Also Microsoft offers support services as consultation, training and certification for developers [14][15]. Since these organizations serve a huge data resources based on Cloud Computing, it needs to be reliable and ease of access to the user moving steps towards the Quality of Service. Quality of service also depends on the usage of resources in different environment that can be categorized as:

1) Private Cloud Computing $\rightarrow$ It is the virtualization of resources used for single organization and provide virtual environment that can be internally or externally hosted.

2) Community Cloud Computing $\rightarrow$ In community cloud computing, virtual environment resources are shared by several organizations and are externally hosted. Also it may be internally hosted by one organization.

3) Public Cloud Computing $\rightarrow$ In public cloud computing, resources and services are provisioned for open use for the public by particular organization that also hosts the service.

4) Hybrid Cloud computing $\rightarrow$ This type of cloud computing is composition of two or more cloud that remain unique entities but are bound together, offering the benefits of multiple deployment models that can be internally and externally hosted [16] .

\subsection{Benefits of Cloud Computing}

Self-Service $\rightarrow$ Cloud Computing provide a benefit of selfservice that allow user to launch application without any cloud service provider. It also designs how the service will be requested from the self-service web- based portal and designs the request fulfillment process, where quality of service can better defined by filtering methods [17].

On-demand $\rightarrow$ Cloud Computing provide the services as per the request of the user that automatically fulfill the process of request. 


\section{International Journal of Science and Research (IJSR) \\ ISSN (Online): 2319-7064}

Index Copernicus Value (2015): 78.96 | Impact Factor (2015): 6.391

Elastic capacity $\rightarrow$ Cloud computing also provides the feature of elasticity that enhance the feature with ability of a system to increase the workload on its current and additional dynamically added on demand hardware resources. Due to virtualization scalability of data center networks are improved [18].

High availability and resiliency $\rightarrow$ It provides the ease and high availability of resources and services to the cloud computer users. In other words it can be said that Cloud Computing can be so designed that the service status can be monitored effectively.

Pay as you go $\rightarrow$ Cloud Computing develops the servicebased cost model and provides cost efficient services so that the user has to pay only for the services they need based on their requirements. Unit price is so defined and designed to induct metering into the services so as to measure consumption.

\subsection{Cloud Deployment Models}

The Cloud represents an evolution in distributed computing that takes advantage of technology as virtualization. Different types of deployment models are as follow:

1) SaaS (software as a service) - This model enables users to to access cloud provider's applications running on a cloud infrastructure. The applications are accessible from various client devices through a thin client interface such as web browser. Common platforms providing software as a service are Salesforce.com, Google Apps and Google Mail.

2) PaaS (platform as a service) - The model enables the users to deploy applications onto the Cloud to be run on preferred development environment or programming language supported by the Cloud. The application developers are freed from the task of installation of Integrated Development Environment(IDE) and focus on the application code. Standard platforms facilitating this model are Amazon Elastic Compute Cloud (EC3), Force.com, and Google AppEngine.

3) IaaS (infrastructure as a service) - In this model the user has the facility for provisioning processing, storage, networks and other computing resources and to deploy and run arbitrary software which can include operating systems and applications. Amazon Private Cloud is the most common IaaS solution to cloud users.

\section{QoS in Grid Computing And Cloud Computing}

Cloud Computing being the current stage in the evolution of on demand information technology services and products within the Distributed Systems and draws heavily on the principles and base of Grid and Utility Computing [19, 20]. In any technology guarantees need to be in place that commit a certain level of performance and involves resources reservation control and monitoring mechanisms for service requirement. There has been much confusion over the term Cloud Computing due to its relative infancy within computer science, its extensive generalized use by industry and the lack of consensus on what a Cloud really is. Today the dynamics of the Internet economy has changed and the recreation of ecommerce as a new source and technology growth within businesses has increased. The following techniques guaranty the QoS that are highly related to Cloud Computing [21].

\section{Resource Management}

Grid Computing gave the concept of Resource management that encompasses the dynamic allocation of tasks to computational resource and requires a scheduler that enhance the performance. QoS is enabled in Grids which is enhanced in cloud computing by the scheduling of tasks [22], which guarantees that resource requirements of an application that are strictly supported, but resources are not over provision. Sequences of tasks are represented as workflows, directed graphs comprised of precedent constrained nodes, which each represent the specific ordered invocation of a service on computation resources to process a given task.

Moving towards Cloud Computing reducing these complexities various algorithms are developed monitoring tools, path scheduling and are essential in ascertaining the availability of resources and providing feedback to schedulers [23], where performance is characterized by the amount of useful work accomplished in comparison to the time and resources used. Monitoring tools are also essential in providing fault tolerance and the migration of tasks $[24,25$, 26].

\section{Service Level Agreement Standardization}

The importance of Service Level Agreements (SLAs) guarantees the reliability of the resources and services provided to the users. The Web Services Agreement is one such standardization by the Open Grid Forum.

The specification consists of three parts which involves the following:

- A schema for specifying an agreement.

- A schema for specifying an agreement template.

- A set of operations for managing an agreements life-cycle.

The life-cycle model does not accommodate the dynamic nature of the Grid economy, providing facilities to negotiate and renegotiate an agreement. The current research in QoS within Grid is concentrated on this problem. Another cutting edge topic of research surrounding QoS in Grid Computing is solving problems related to risk assessment and dependability of service providers and is being tackled by projects such as Assess Grid. The Cloud Computing also finds issues in providing guarantees within two parties and need to be improved for sort. Standardizing aspects of SLAs improves the clarity and increases the understanding of SLAs for cloud services in the market in particular by highlighting and providing information on the concepts usually covered by SLAs [27].

\section{Virtualization}

Cloud computing system uses the concept of virtualization that is an important development in Computer Science an efficient access of one or more complete computer systems, data centres, virtual machines and other hardware resources. It provides very ease and efficient access of data over the 


\section{International Journal of Science and Research (IJSR) \\ ISSN (Online): 2319-7064}

Index Copernicus Value (2015): 78.96 | Impact Factor (2015): 6.391

network. Virtualization of resources has improved testing and installation of different version of operating system, running with virtual configuration when there is increase in memory, processors or input output devices. Virtualization also provides hardware enhancement to the configuration without re-coding that already exist. It provides high degree of reliability and security for the applications on demand. Earlier when virtualization was proposed for the design of virtual machines i.e. virtualizable architectures that had few disadvantages that either they supported new complex operating systems directly on the virtual machines or they have been unable to avoid all flaws associated with virtual machine support. Then with the quality improvement hardware virtualizer avoided the weaknesses of previous designs while at the same time incorporating their strong points.

Virtualization in Cloud Computing combines the following design that is divided as hardware, hypervisor, virtual machines and Cloud Service Provider Management Layer. In virtualization technology, hypervisor is a program that manages multiple OS or multiple instances of the same operating system on a single machine. It is also known as virtual machine monitor (VMM) or virtualization monitors as it manages the system's resources to allocate what each operating system requires, where a hypervisor runs on a system indicated as host machine. Each virtual machine has its own processor, memory and other hardware resources, where virtual machine (VM) is an operating system or application environment that is installed on software which imitates dedicated hardware.

The Virtual Machines requests the Cloud Service Provider for the services that is managed by CSP management layer. Thus cloud provider offers some component of cloud computing i.e. Infrastructure as a Service (IaaS), Software as a Service (SaaS) or Platform as a Service (PaaS) to other businesses, organizations or individuals.

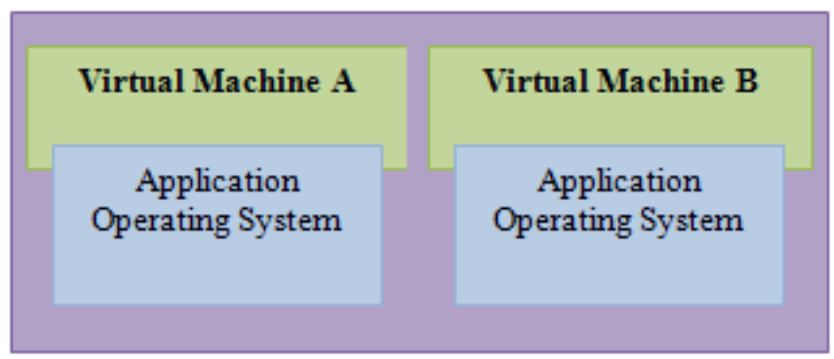

Figure 1: Virtualization in Cloud

Virtualization technology provides a better effort to maintain the resources offered to the users which have the following attributes as multi-tenancy, highly scalableand elastic, selfprovisioned, pay-per-use price model [28], where multitenancy is data and application in a VM share the same physical resources with other VM which can have a malicious behavior [29][30]. The impact of virtualization in the commercial environment can be best seen in the amazon ec2 data center that has optimized the cost of services [31].

One smart way to view "cloud" is as a new evolution of IT service delivery from a remote location, involving multitenant environments enabled by virtualization as compared to grid computing [32]. Cloud architecture is underpinned and enabled by commodity hardware on top of which virtual machines are provisioned and offered to consumers. Elasticity is an important component of cloud computing, but it is both a feature and a natural result of the main drivers of cloud computing: economies of scale, improved efficiency and speed. As said, the lack of a settled and universal "cloud" definition means businesses need to make their cloud services work for them. A business needs to define its goals and objectives for a cloud computing offering as well as the benefits sought, and look for providers that will maximize those advantages. Simply being located in the cloud is not enough; nor should the possibility of cost savings be a permanent, compelling reason to adopt. Even though cloud is a new delivery method, it must conform to the old rules of producing superior business.

\section{Related Work}

A continuous work is being done to improve Quality of Service in Cloud Computing. In this section overview of research is being presented. Although a lot of work has been done by the researchers in the field of Quality of Service of Cloud Computing, still there are challenges that is to be improved in reference to various parameters of Quality of Service[33]. Various approaches are made for the better performance of the Services and to improve the Quality of Service in Cloud Computing that will be studied and developed further [34]. Thus Cloud Computing provides methods and techniques to these challenges that includes virtualization of resources in all aspects for the ease of data access on distributed network, securing data on cloud computing, exploring the current situations of quality improvement of data availability and restoration, cost optimality on cloud computing, improving performance measure and assurance, ease for usability at the end users [35].

As it is popularly said that "Quality matters a lot than Quantity", and when we talk about the data quality stored in large number it defines various parameters as performance, availability and service quality[. These parameters can further be specified as restoration of data, load balancing, reliability, data management and so on which has a vital role in improving Quality of Service. Quality of Service has its own importance in every aspect for better improvement of performance. As a consequence for further Cloud Computing research we identify a large number of problems that need to be addressed. Cloud computing is changing more and more services on Internet[36][37][38]. The Quality of Service (QoS) will decrease if the data centre is far from the end users. In such a multi-cloud environment, applications based on cloud should make choices of how to use these resources. Security in cloud computing is also very important. Lots of works have been done to resolve this problem. In multicloud, security problem is more important and difficult[39]. With such a standard security management, cooperation in multi-cloud providers can be achieved[40]. The cost of VM is dynamically changing with the application requirements. Less VMs than needed leads to a high resource utilization. More VMs than needed will cause a waste of resource. The standard of the needed number is based on QoS $[41][42][43][44]$. 


\section{International Journal of Science and Research (IJSR) \\ ISSN (Online): 2319-7064}

Index Copernicus Value (2015): 78.96 | Impact Factor (2015): 6.391

\section{QoS Characterstics in Cloud Computing}

Quality of Service parameters as performance, reliability, cost, response time, security etc builds an important characteristics in Cloud Computing [45][46]. Cloud computing is rapidly developing and becoming more and more attractive for Small and Medium Enterprises (SME) as well as large organizations. Low cost, high efficiency and scalability are very significant in the environment of large data stored over the network, which is becoming a trend. In cloud computing, environment cost is a critical measure for both providers with an objective that the services provided to the users are low in cost as well as the profit earned by the service providers is maximized. For fulfilling some conflictive objectives while ensuring QoS efficient algorithm need to be developed [47][48].

Researchers have suggested that Cloud computing is an operation model that integrates many technological advancements of the last decade such as virtualization, web services, and SLA management for enterprise applications. Therefore it has been identified that characterizing cloud systems will require use of diverse modeling techniques to scope with such technological heterogeneity. The QoS modeling literature is extensive, making it difficult to have a comprehensive view of the available techniques and their current applications to cloud computing problems. Still a lot of work is to be done in this direction to make the cloud computing more effective and optimized and ease of access for the end users by various techniques [49].

\section{QoS Characteristics in Cloud Computing:}

Implementation of Cloud Computing in the fast growing technologies have improved a lot in the direction of quality of service. Quality of service in Cloud Computing is a wide area to be worked on and include number of parameters as follows:

Accountability $\rightarrow$ This group of QoS attributes is used to measure various Cloud provider specific characteristics. This is important to build trust of a customer on any Cloud provider. No organization will want to deploy its applications and store their critical data in a place where their is no accountability of security exposures and compliance. Functions critical to accountability, which SMI considers when measuring and scoring services, include auditability, compliance, data ownership, provider ethicality, sustainability etc.

Agility $\rightarrow$ The most important advantage of Cloud computing is that it adds to the agility of an organization. The organization can expand and change quickly without much expenditure. Agility in SMI is measured as a rate of change metric, showing how quickly new capabilities are integrated into IT as needed by the business. When considering a Cloud service's agility, organizations want to understand whether the service is elastic, portable, adaptable and flexible.

Cost $\rightarrow$ The first question that arises in the mind of organizations before switching to Cloud computing is that whether it is cost-effective or not. Therefore, cost is clearly one of the vital attributes for IT and the business. Cost tends to be the single most quantifiable metric today, but it is important to express cost in the characteristics which are relevant to a particular business organization. On-Demand cost can be collected only for the usage of resources. It does not include any minimum charges. And in this ranking scheme on demand cost can be calculated by VM cost, data cost and storage cost. Reserving the resources may reduce the cost compared to on demand cost. But in includes onetime registration cost. Therefore reservation cost can be calculated.

Performance $\rightarrow$ There are many different solutions offered by Cloud providers addressing the IT needs of different organizations. Each solution has different performance in terms of functionality, service response time, accuracy, stability, interoperability, suitability etc. These organizations need to understand through these properties how well their applications will perform on the different Clouds and whether these deployments meet their expectations [47][48].

Assurance $\rightarrow$ This characteristic indicates the likelihood of a Cloud service that it will perform as expected or promised in the SLA. Every organization looks to expand their business and provide better services to their customers. Therefore availability, reliability, resiliency and service stability become an important factor for them before they decide switching to Cloud services[49][50].

Security and Privacy $\rightarrow$ Data protection and privacy are the important concerns of nearly every organization. Hosting data in other organizations control is always a critical issue which require stringent security policies employed by Cloud providers[51]. For instance, Financial organizations generally require high compliance regulations involving data integrity and privacy. Security and Privacy is also multi-dimensional in nature and include many attributes such as privacy, confidentiality, data loss and integrity.

Usability $\rightarrow$ For fast usage of Cloud services, the usability plays an important role. The more easy to use and learn a Cloud service is, more faster an organization can switch to Cloud services. The usability of a Cloud service can depend on multiple factors such as accessibility, installability, learnability, operatibility etc.

Further for the better performance, the service response time should be minimum so that fast service can be made available for usage. Stability is defined as the variability in the performance of a service. For storage, it is the variance in average read and Stability is defined as the variability in the performance of a service[52]. Accuracy of the service can be defined as the degree of proximity to the user's actual values to the expected values. Availability is defined as the percentage of time a customer can access the service. Interoperability is the ability of a service to interact with other services offered by same provider or other providers. Reliability can be defined as how a service operates without failure during a given time and condition. Confidentiality is an integral component of security. It ensures that the information stored on the cloud is protected against the unintended or unauthorized access. It is also defined as the percentage of authorized access. Data integrity is the assurance that the information kept on the cloud can only be 


\section{International Journal of Science and Research (IJSR) \\ ISSN (Online): 2319-7064}

Index Copernicus Value (2015): 78.96 | Impact Factor (2015): 6.391

accessed and modified by the authorized one. It is also defined as the accuracy and consistency of the data. Privacy is defined as the state or condition of being free from being observed or disturbed by others. That is the information kept on the cloud should not be disturbed by others. Learnability is the capability of the service to enable the customers to learn how to use the service. Installability can be defined as the capability of the service to be installed in a specific environment. Easiness: It is the capability of the customers of being understood. That is how much the customers are aware about the service functionality. Adaptability is the ability of the service provider to adjust changes in the service based on the customer request and improves the usability of the software[53][54]. Flexibility can be defined as the capability of being changed or adapted repeatedly without much expenditure. Portability can be defined as the capability of running over two or more services. Cloud computing environment has heterogeneous data centers where these QoS metrics[55] can be improved by control measures of service providers[56].

\section{Conclusion and Future Work}

Virtualization of resources have provided a better performance of services and fast availability of data through cloud computing techniques. In the paper authors have presented a quantitative approach to QoS in Cloud Computing that includes the characteristics and matrices of QoS in Cloud Computing. A review has been done in the direction to improve the Quality of Service parameters on cloud computing. Many methods and techniques are used for better response on the parameters that has vital role to enhance Quality of Service on cloud computing. The parameters need to be improved are the response time, cost optimality and power consumption of the data stored on cloud computing over the network. Future Work shall focus on improving the fast availability of data over the network to provide cost optimal services and improving power consumption, to optimize Quality of Services on Cloud Computing and other techniques to improve parameters of QoS in Cloud Computing. The authors are of the view that there is need to improve the quality of services on various measures, where security of data on cloud computing plays an important role to keep the data reliable without any threat.

\section{References}

[1] Mahmoud Naghibzadeh, Saied Abrishami, Dick Epema, "Cost- driven Scheduling of Grid Workflows Using Partial Critical Paths" IEEE Transactions on Parallel \& Distributed Systems, vol. 23 ISSN: 1045-9219 pp: 1400-1414, 08 - Aug 2012.

[2] Lena Mashayekhy, Mahyar Movahed Nejad, and Daniel Grosu, "Physical Machine Resource Management in Clouds: A Mechanism Design Approach", IEEE Transactions On Cloud Computing, Vol. 3, No. 3, JulySeptember 2015.

[3] Bhanu Sharma, Ruppa K. Thulasiram, Parimala Thulasiraman, and Rajkumar Buyya "Clabacus: A RiskAdjusted Cloud Resources Pricing Model Using Financial Option Theory", IEEE Transactions On Cloud Computing, Vol. 3, No. 3, July-September 2015.

[4] R. Buyya, C. S. Yeo, S. Venugopal, J. Broberg, and I.
Brandic, "Cloud computing and emerging IT platforms: Vision, hype, and reality for delivering computing as the 5th utility, Future Generation Computer Systems", vol. 25, no. 6, pp. 599-616, 2009.

[5] Ardagna D, Panicucci B, Trubian M, Zhang L (2012) "Energy-aware autonomic resource allocation in multitier virtualized environments." IEEE Trans Serv Comput 5(1):2-19Publisher Full Text .

[6] M. A. AlZain, E. Pardede, B. Soh, and J. A. Thom, "Cloud computing security: From single to multi-clouds, presented at System Science (HICSS)", Hawaii International Conference on. IEEE, Maui, HI, USA, 2012.

[7] Z. Chen, F. Han, J. Cao, and S. Chen, "Cloud computing based forensic analysis for collaborative network security management system", Tsinghua Science and Technology, vol. 18, no. 1, pp. 40-50, 2013.

[8] Nirnay Ghosh, Soumya K. Ghosh, and Sajal K. Das," SelCSP: A Framework to Facilitate Selection of Cloud Service Providers", IEEE Transactions On Cloud Computing, Vol. 3, No. 1, January-March 2015.

[9] Zibin Zheng, Xinmaio Wu, Yilei Zhang, Michael R. Lyu, and Jianmin Wang," QoS Ranking Prediction for Cloud Services", IEEE Transactions On Parallel And Distributed Systems, 2012.

[10] A. Iosup, S. Ostermann, N. Yigitbasi, R. Prodan, T. Fahringer, and D. Epema, "Performance analysis of cloud computing services for many-tasks scientific computing," IEEE Trans. Parallel Distributed System, vol. 22, pp. 931-945, June 2011.

[11]H. Khazaei, J. Misic, and V. B. Misic, "Performance analysis of cloud computing centers using $\mathrm{m} / \mathrm{g} / \mathrm{m} / \mathrm{m}+\mathrm{r}$ queuing systems," IEEE Trans. Parallel Distributed System, vol. 23, no. 5, pp. 936-943, May 2012.

[12] G. Linden, B. Smith, and J. York, "Amazon.com recommendations: Item-to-item collaborative filtering," IEEE Internet Computing, vol. 7, no. 1, pp. 76-80, 2003.

[13]S. Hazelhurst."Scientific Computing Using Virtual Highperformance Computing: A Case Study Using the Amazon Elastic Computing Cloud", Proceeding 2008 Annu. Research Conf. South African Institute of Computer Scientists and Information Technologists (SAICSIT 2008), ACM Press, 2008, pp. 94-103

[14]Z. Li, L. O’Brien, R. Cai, and H. Zhang. "Towards a Taxonomy of Performance Evaluation of Commercial Cloud Services", Proceeding 5th Int. Conf. Cloud Computing (IEEE CLOUD 2012), IEEE Computer Society, 2012.

[15] M. Armbrust, A. Fox, R. Griffith, A. D. Joseph, R. Katz, A. Konwinski, and M. Zaharia, "A view of cloud computing, Communications of the ACM", vol. 53, no. 4, pp. 50-58, 2010.

[16]R. Burke, "Hybrid recommender systems: Survey and experiments," User Modeling and User-Adapted Interaction, vol. 12, no. 4, pp. 331-370, 2002.

[17] J. Wu, L. Chen, Y. Feng, Z. Zheng, M. Zhou, and Z. $\mathrm{Wu}$, "Predicting qos for service selection by neighborhood-based collaborative filtering," IEEE Transactions on System, Man, and Cybernetics, Part A, to appear.

[18] Meng X, Pappas V, Zhang L "Improving the scalability of data center networks with traffic-aware virtual machine placement." In: Proceedings of the 29th 


\section{International Journal of Science and Research (IJSR) \\ ISSN (Online): 2319-7064}

Index Copernicus Value (2015): 78.96 | Impact Factor (2015): 6.391

Conference on Information Communications, INFOCOM'10, 1154-1162, San Diego, CA, USA., 2010.

[19] Danilo Ardagna, Giuliano Casale, Michele Ciavotta, Juan F Pérez and Weikun Wang, "Quality-of-service in cloud computing: modeling techniques and their applications", Journal of Internet Services and Applications, 2014.

[20]D. Warneke and O. Kao, "Exploiting Dynamic Resource Allocation for Efficient Parallel Data Processing in the Cloud," IEEE Trans. on Parallel and Distributed Systems, Vol.22, No.6, pp.985-997, 2011.221

[21]Z. Zheng and M. R. Lyu, "WS-DREAM: A distributed reliability assessment mechanism for Web services," in Proc. 38th Int'l Conf. Dependable Systems and Networks (DSN'08), 2008, pp. 392-397.

[22] Maria Alejandra Rodriguez, Rajkumar Buyya," Deadline Based Resource Provisioning and Scheduling Algorithm for Scientific Workflows on Clouds", IEEE Transactions On Cloud Computing, Vol. 2, No. 2, April-June 2014.

[23] Van den, Bossche R, Vanmechelen K, Broeckhove J "Cost-optimal scheduling in hybrid iaas clouds for deadline constrained workloads." In: Proceedings of the 2010 IEEE 3rd International Conference on Cloud Computing, CLOUD'10, 228-235, Miami, FL, USA.,2010.

[24]Chien-An Chen, Myounggyu Won, Radu Stoleru, "Energy-Efficient Fault-Tolerant Data Storage and Processing in Mobile Cloud", IEEE Transactions On Cloud Computing, Vol. 3, No. 1, January-March 2015.

[25]P. Sempolinski and D. Thain, "A Comparison and Critique of Eucalyptus, OpenNebula and Nimbus," Proceedings of the 2nd IEEE International Conference on Cloud Computing Technology and Science, pp.417426, 2010.

[26] Venkata Josyula, Malcolm Orr, Greg Page (2011), "Cloud Computing: Automating the Virtualized Data Center". Journal of Emerging Technology and Advanced Engineering Website, Volume 4, 2014.

[27] Brussels, "Cloud Service Level Agreement Standardisation Guidelines" 2014.

[28] Ye Z, Bouguettaya A, Zhou X " QoS-aware cloud service composition based on economic models." In: Proceedings of the 10th International Conference on Service-Oriented Computing, ICSOC'12, 111-126, Shanghai, China.,2012.

[29] Menascé DA, Casalicchio E, Dubey VK (2010) On optimal service selection in service oriented architectures. Perform Eval 67(8):659675 Publisher Full Text

[30] Longo F, Ghosh R, Naik VK, Trivedi KS "A scalable availability model for Infrastructure-as-a-Service cloud." In: Proceedings of the 2011 IEEE/IFIP 41st International Conference on Dependable Systems Networks, DSN 2011, 335-346, Hong Kong, China., 2011.

[31] Wang G, Ng TSE "The impact of virtualization on network performance of amazon ec2 data center." In: Proceedings of the 29th Conference on Information Communications, INFOCOM'10, 1163-1171, San Diego, CA, USA, 2010.

[32] I. Foster, Y. Zhao, I. Raicu, and S. Lu, Cloud computing and grid computing 360-degree compared, presented at
Grid Computing Environments Workshop, Austin, TX, USA, 2008.

[33] N.Ani Brown Mary, K.Jayapriya, Vin Solutions, "An Extensive Survey on QoS in Cloud computing."

[34] Abdelzahir Abdelmaboud, Dayang N.A. Jawawi, Imran Ghani, Abubakar Elsafi, Barbara Kitchenham, "Quality of service approaches in cloud computing: A systematic mapping study" Journal of Internet Services and Applications, 2014.

[35] Mao M, Humphrey M, "A performance study on the VM startup time in the cloud." In: Proceedinngs of the 2012 IEEE Fifth International Conference on Cloud Computing, CLOUD '12, 423-430, Honolulu, HI, USA.

[36] Buyya et al., "Cloud Computing and Emerging IT Platforms: Vision, Hype, and Reality for Delivering Computing as the 5th Utility," Future Generation Computer Systems, vol. 25, no. 6, pp. 599-616, 2009.

[37] J. Varia, Cloud Computing: Principles and Paradigms. Wiley Press, 2011, ch. 18: Best Practices in Architecting Cloud Applications in the AWS Cloud, pp. 459-490.

[38] M.D. Dikaiakos, D. Katsaros, P. Mehra, G. Pallis and A. Vakali, "Cloud Computing: Distributed Internet Computing for IT and Scientific Research," IEEE Internet Computing, Vol.13, No.5, pp.10-13, 2009. 220

[39]A. Sampaio and N. Mendonca, "Uni4Cloud: An approach based on open standards for deployment and management of multi-cloud applications, in Processdings of the 2nd International Workshop on Software Engineering for Cloud Computing", New York, NY, USA, 2011.

[40] J. Li, J. Chinneck, M. Woodside, M. Litoiu, G. Iszlai, "Performance model driven QoS guarantees and optimization in clouds, presented at Software Engineering Challenges of Cloud Computing", ICSE Workshop on, Vancouver, BC, Canada, 2009.

[41] D. Kondo, B. Javadi, P. Malecot, F. Cappello, and D. P. Anderson, "Cost-benefit analysis of cloud computing versus desktop grids", presented at Parallel \& Distributed Processing IPDPS Rome, Italy, 2009.

[42]D. Yuan, Y. Yang, X. Liu, and J. Chen, "A costeffective strategy for intermediate data storage in scientific cloud workflow systems", presented at Parallel $\&$ Distributed Processing (IPDPS), IEEE International Symposium on, Atlanta, GA, USA, 2010.

[43] J. Cao, K. Hwang, K. Li, and A. Y. Zomaya, "Optimal multiserver configuration for profit maximization in cloud computing", IEEE Trans. Parallel and Distributed Systems, vol. 24, no. 6, pp. 1087-1096, 2013.

[44] J. L. L. Simarro, R. Moreno-Vozmediano, R. S. Montero, and I. M. Llorente, "Dynamic placement of virtual machines for cost optimization in multi-cloud environments", in 2011

[45] John W. Rittinghouse, James F. Ransome (2009), "Cloud Computing: Implementation, Management, and Security

[46] Xingwei Wang, Xueyi Wang, Hao Che, Senior Member, IEEE, Keqin Li, Fellow, IEEE, Min Huang, and Chengxi Gao, "An Intelligent Economic Approach for Dynamic Resource Allocation in Cloud Services", IEEE Transactions On Cloud Computing, Vol. 3, No. 3, JulySeptember 2015.

[47]A. Iosup, N. Yigitbasi, and D. Epema, "On the performance variability of production cloud services," 
Proceedings of IEEE/ACM International Symposium on Cluster, Cloud, and Grid Computing, CA, USA, 2011.

[48] A. Iosup, S. Ostermann, N. Yigitbasi, R. Prodan, T. Fahringer, and D. Epema, "Performance analysis of cloud computing services for many-tasks scientific computing," IEEE Transactions on Parallel and Distributed Systems, vol. 22, no. 6, pp. 931-945, 2011.

[49] B. Ahlgren, P.A. Aranda, P. Chemouil, S. Oueslati, L.M. Correia, H. Karl, M. Sollner and A. Welin, "Content, Connectivity and Cloud: Ingredients for the Network of the Future," IEEE Communications Magazine, Vol.49, No.7, pp.62-70, 2011.

[50] V. Tran, H. Tsuji, and R. Masuda, "A new qos ontology and its qos-based ranking algorithm for web services," Simulation Modelling Practice and Theory, vol. 17, no. 8, pp. 1378-1398, 2009.

[51] Srinivasan, S. (2014), "Security, Trust, and Regulatory Aspects of Cloud Computing in Business Enviorment."

[52] K. J. arvelin and J. Kekalainen, "Cumulated gain-based evaluation of IR techniques," ACM Transactions on Information Systems, vol. 20, no. 4, pp. 422-446, 2002.

[53] Padala P, Shin KG, Zhu X, Uysal M, Wang Z, Singhal S, Merchant A, Salem K,"Adaptive control of virtualized resources in utility computing environments." In: Proceedings of the 2Nd ACM SIGOPS/EuroSys European Conference on Computer Systems 2007, EuroSys'07, 289-302, Lisbon, Portugal, 2007.

[54] A. Li, X. Yang, S. Kandula, and M. Zhang, "Cloudemp: comparing public cloud providers," in Proceedings of the 10th Annual Conference on Internet Measurement, Melbourne, Australia, 2010.

[55] Delimitrou C, Bambos N, Kozyrakis C "QoS-aware admission control in heterogeneous datacenters." In: Proceedings of the 2013 10th International Conference on Autonomic Computing, ICAC ÃŢ13, 291-296, San Jose, CA, USA, 2013.

[56] Amid Khatibi, Seyyed Mohsen Hashemi, "QoS Metrics for Cloud Computing Services Evaluation." 